\title{
Potts model on directed complex networks
}

\begin{abstract}
It is known from the literature that the Ising and Potts models in one dimension $(d=1)$ do not present phase transitions at finite temperature $T$, for any number of states $q$. However, in two dimension $(d=2$, ) there are a second-order phase transition and a first-order transition for $\mathrm{q} \leq 4$ and $\mathrm{q} \geq 5$, respectively. On directed Barabási-Albert networks, the Potts model with $q=2$ states (Ising model) no presents a phase transition. On the other hand, the Potts with $\mathrm{q}=3$ presents a first-order phase transition well defined on these networks. This behavior is different from the Potts model with $q=2$ and 3 states on a square lattice where the phase transition is of the second-order. Here, we will briefly discuss the critical behavior of the Potts model on directed complex networks as the Barabási-Albert network also known as free scale networks.
\end{abstract}

Keywords: potts, networks, spins, ising model, scale networks, square lattice, two dimension, temperature, elementary models, statistical physics
Volume 3 Issue 4 - 2019

\section{FWS Lima}

Departamento de Física, Universidade Federal do Piauí, Brazil

Correspondence: FWS Lima, Departamento de Física, Dietrich Stauffer Computational Physics Lab, Universidade Federal do Piauí, 64049-550,Teresina - PI, Brazil, Email fwslima@gmail.com

Received: August 08, 2019 | Published: August 29, 2019

\section{Introduction}

A large amount of information about the behavior of real, physical systems can be described using simple models supplied from statistical physics. This is occur due to the phenomenon of universality near a second-order phase transition where the behavior critical of the system only depends on a few parameters like the dimensionality and global symmetries of the system. Because of this, elementary models like the Ising model remain of great importance. The Potts model is a generalization of the simplest Ising model in statistical mechanics for systems with more than two opinions (yes or not).

The Potts model in two-dimension (2D) present phase transition well defined at finite temperature $T$, for any number of states $q>1$. However, in 2D the phase transition presents a second-order phase transition for $q \leq 4$ and a first-order transition for

and $q \geq 5$, respectivelly. ${ }^{1}$

Édio et al., ${ }^{2}$ studied the Potts model on a directed one-dimension on Small-World network. They found that for $q=3$ the Potts model presents a second-order phase transition for rewiring probability $p$ in the range of $0<p<0.15$ and first-order phase transition for $1>p>0.15$. In the particular case of $p=0.1$ the critical exponent ratios $1 / \nu, \beta / v$ and $\gamma / v$ fall into a different universality class than the model on a regular two-dimensional lattice neglecting or not corrections to scaling. For the case $q=8$ our results indicate only first-order phase transition for $p>0$. One possible explanation for this behavior is the influence of long range interactions that occur in the presence of $p$ directed bonds and also the number of the Potts model states on the directed SW network.

Lima $^{3,4}$ studied the q=3 states ferromagnetic Potts model on ErdösRènyí random graphs and Solomon networks. Unlike the results presented for the Potts model on a square lattice, their results showed that this model presents only a first-order phase transition. The Ising model no presents phase transition on directed Barabási-Albert (BA) network. ${ }^{5}$ Lima $^{6}$ studied the Potts model on directed BA networks.
The results show that for the Potts model with $q \geq 3$ states presents again only a first-order phase transition.

\section{Potts model}

To study the behavior critical of the Potts model, we need to evolve the hamiltonian this model defined below

$H=-J \sum_{i=1}^{N} E_{i}$,

with

$$
E_{i}=\sum_{j=1}^{k} \ddot{a}_{i} S_{j}
$$

where the sum is carried out over the $k$ neighbors of site $i$. In the above equation $J$ is the exchange coupling. The energy per spin is given by,

$$
e=\frac{E_{i}}{N}
$$

and the magnetization,

$$
M=\frac{\left(q \cdot \max \left[n_{i}\right]-N\right)}{q-1}
$$

where the magzation per spins $\mathrm{m}=\mathrm{M} / \mathrm{N}$ was evaluated. Here, $n_{i} \leq N$ denotes the number of spins with "orientation" $i=1, \ldots, q$.

\section{Conclusion}

The two-dimensional Potts model presents two phase transition types, one of second-order and one of first-order. For $q \leq 4$ the transition is of second-order and for larger $q$ we have a first order phase transition. The reason for this behavior so far has not been explained in the literature.

Here, we have shown that by considering the $q$-states 
ferromagnetic Potts model on directed Erdös-Rènyí random graphs, Solomon, and BA networks, there is only a first-order phase transition. Unfortunately, so far, we have no plausible reason to justify this behavior of the Potts model on the directed networks mentioned here.

\section{Acknowledgements}

The author would like to thank the Brazilian agencies CNPq and Capes.

\section{Conflicts of Interest}

The author states that there is no conflict of interest.

\section{References}

1. DP Landau, K Binder. A Guide to Monte Carlo Simulations in Statistical Physics. Second Edition, Cambridge University Press. 2005. p. 1-489.

2. Édio O Aquino, FWS Lima, Ascânio D Araújo, et al. Potts Model in
One-Dimension on Directed Small-World Networks. Journal Statistical Physics. 2018;171(6):1112-1121.

3. FWS Lima. Potts Model With $\mathrm{q}=3$ States on Directed Erdös-Rènyi Random Graphs. Phys Astron Int J. 2017;1 (6):1-3.

4. FWS Lima. Potts model with $\mathrm{q}=3$ states on solomon networks. Physics \& Astronomy International Journal. 2018;2(2):61-63.

5. Munner A Sumour, MM Shabat. Monte carlo simulation of ising model on directed barabasi-albert network. Int. J. Mod. Phys. 2005;16(4):585-589.

6. FWS Lima. Potts Model with Q States on Directed Barabási-Albert Networks. Communications in Computational Physics. 2007;2(3):522529. 